\title{
Voltage and Length-Dependent Phase Diagram of the Electronic Transport in Carbon Nanotubes
}

\author{
Per Sundqvist, ${ }^{\dagger}$ Francisco J. Garcia-Vidal, ${ }^{*, \dagger}$ Fernando Flores, ${ }^{\dagger}$ \\ Miriam Moreno-Moreno, ${ }^{\ddagger}$ Cristina Gómez-Navarro, ${ }^{\S}$ Joseph Scott Bunch," and \\ Julio Gómez-Herreroł
}

\begin{abstract}
Departamento de Física Teórica de la Materia Condensada, Universidad Autónoma de Madrid, E-28049 Madrid, Spain, Departamento de Física de la Materia Condensada, Universidad Autónoma de Madrid, E-28049 Madrid, Spain, Nanoscale Science Department, Max-Planck-Institute for Solid State Research, D-70569 Stuttgart, Germany, and Clark Hall Lassp, Cornell University, Ithaca, New York 14853
\end{abstract}

Received March 30, 2007; Revised Manuscript Received June 27, 2007

\begin{abstract}
In this work, we report experimental data on the evolution of the resistance with applied voltage in nonsuspended single-walled carbon nanotubes (SWNTs) of lengths ranging from $100 \mathrm{~nm}$ up to $6 \mu \mathrm{m}$. At low bias, the differential resistance as a function of length is well described by a linear fitting. At high biases, this magnitude first saturates and then decreases for nanotubes longer than $1 \mu \mathrm{m}$. We also present Monte Carlo numerical simulations for the one-dimensional Boltzmann's equation, describing how the electrons propagate along the tube and how they interact with acoustic and optical phonons. Our theoretical results show a remarkable agreement with the experimental differential resistance, allowing us to give a detailed description of the electron distribution function and the chemical potential along the nanotube. Finally, we present experimental results on the transition from Anderson localization at low bias to high diffusive regime at high bias in defected SWNTs. This result is combined with those of defect-free SWNTs to present a general landscape of the electronic transport in carbon nanotubes.
\end{abstract}

Carbon nanotubes are the cornerstone in molecular electronics. Since their discovery in $1991,{ }^{1}$ they have been used as a model system to study electronic transport at the nanoscale. $^{2-7}$ At low bias, defect-free metallic single-walled carbon nanotubes (SWNTs) are quasiballistic conductors presenting a very weak interaction between electrons and acoustic phonons. For high biases (above $0.2-0.3 \mathrm{~V}$ ), strong scattering of electrons with optical phonons destroys their phase coherence, resulting in a highly diffusive electronic transport characterized by a linear dependence of the resistance versus length. ${ }^{8-11}$ To investigate the evolution of the electronic transport with the length and bias for long $(L>1 \mu \mathrm{m})$ nonsuspended SWNTs have been prepared on a silicon substrate sample with an oxide layer of $500 \mathrm{~nm}$. The nanotubes are contacted to a gold electrode, and then a current versus voltage $(I V)$ curve is acquired along their

\footnotetext{
* Corresponding author. E-mail: fj.garcia@uam.es.

† Departamento de Física Teórica de la Materia Condensada, Universidad Autónoma de Madrid.

$\stackrel{\dagger}{\dagger}$ Departamento de Física de la Materia Condensada, Universidad Autónoma de Madrid.

$\S$ Nanoscale Science Department, Max-Planck-Institute for Solid State Research.

"Clark Hall Lassp, Cornell University.
}

length by using a conductance atomic force microscope (AFM) from Nanotec Electronica. ${ }^{12}$ As the tip pushes the nanotube, the optimum contact force is reached ${ }^{13}$ and then an $I V$ (see inset in Figure 1) is acquired for biases $\pm 2.2 \mathrm{~V}$. By calculating the derivative of the $I V$ curves, the differential resistance, $R=\mathrm{d} V / \mathrm{d} I$, is numerically obtained as a function of the voltage and the distance between both electrodes (i.e., the length of the nanotube). ${ }^{14}$ For the present work, only metallic SWNTs are considered.

Figure 1 shows the low voltage (approximately $0.1 \mathrm{~V}$ ) differential resistance as a function of the electrodes distance $(L)$ for two different types of SWNTs. The square dots correspond to an individual HipCo-type SWNT from Carbon Nanotechnologies Inc., prepared by suspending them in a surfactant solution and treated with ultrasound to remove bundles. These SWNTs were then adsorbed on a silicon oxide substrate by drop-casting. The energy introduced by the ultrasound bath disperses the bundles but, in addition, it creates structural defects along the SWNTs. The rest of the data corresponds to seven different metallic SWNTs directly grown on the surface by standard chemical vapor deposition (CVD). ${ }^{15}$ The resistance versus length relationship for HipCo nanotubes follows an exponential law (see Figure 1), which 


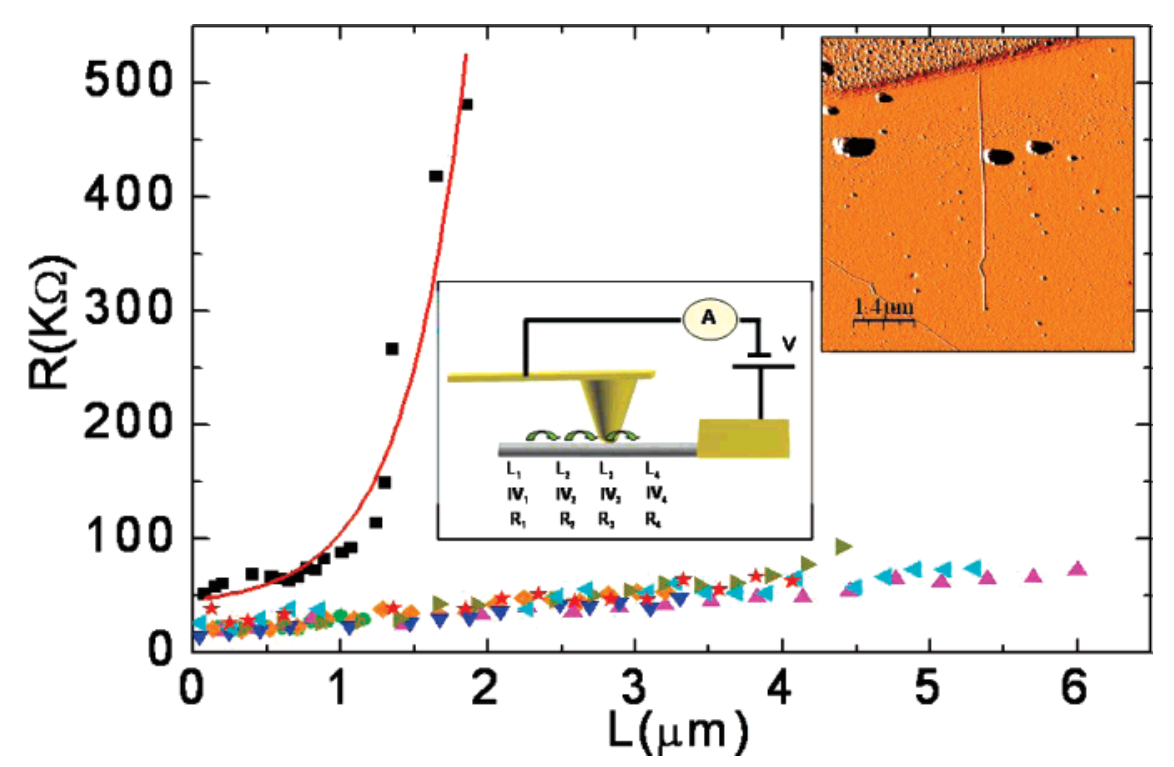

Figure 1. Experimental dependence of the low voltage resistance vs length for CVD-grown SWNTs (triangles and stars) and HipCo SWNTs (squares). The experimental set up is illustrated in the central inset of the figure. A metallic AFM tip is moved along the nanotube probing its electrical conductance as a function of the tip-electrode distance. The upper inset is an AFM image of a long nanotube partially covered with gold. The nanotubes directly grown on surface present low disorder and hence the conductance is quasiballistic; the low increment of the resistance with length is a consequence of the interaction with acoustic phonons and disorder. The HipCo SWNT presents a high density of defects resulting in an electronic transport regime governed by the Anderson localization phenomenon.

is a fingerprint of the appearance of the Anderson localization phenomenon. ${ }^{16}$ As the electrons travel along the nanotube, they scatter elastically with the structural defects of the lattice but their phase is preserved. It has been shown ${ }^{17,18}$ that if the localization length, $L_{0}$, is smaller than the electron mean free path due to scattering with acoustic phonons, defects dominate the low-bias transport in SWNTs, resulting on an exponential increase of the resistance versus length. By fitting $R(L) \propto e^{L / L_{0}}$, we estimate that $L_{0}$ in our HipCo nanotube is around $420 \mathrm{~nm}$. On the other hand, for CVD-grown SWNTs, the plots display a linear dependence of $R$ versus $L$, as expected for an ohmic conductor. It is remarkable that the slopes of the curves (i.e., the linear resistivity at low bias, $\left.\rho_{\text {low }}\right)$ are quite similar for the seven nanotubes considered, presenting an average value $\rho_{\text {low }}=10 \pm 2 \mathrm{k} \Omega / \mu \mathrm{m}$ and a contact resistance of about $18 \pm 6 \mathrm{k} \Omega$. The relationship between the linear resistivity and the electron mean free path due to scattering with acoustic phonons, $\lambda_{\mathrm{ac}}$, is given by $\rho$ $=\left(h / 4 e^{2}\right)\left(1 / \lambda_{\mathrm{ac}}\right)$. By fitting to the experimental data, $\lambda_{\mathrm{ac}}$ in our CVD grown SWNTs is $650 \pm 130 \mathrm{~nm} .{ }^{19}$

Now we consider the high-bias regime $(V>0.3 \mathrm{~V})$, when electrons can excite optical phonons. Panel (b) in Figure 2 is a set of differential conductance curves measured at different distances along a $6 \mu \mathrm{m}$ long nanotube (one of the seven CVD-grown SWNTs studied in Figure 1, triangle dots pointing up). The conductance curves have a maximum near $0 \mathrm{~V}$ and then decay as the inverse of the length for short distances. From this plot, we can extract the dependence of $R$ with $L$ at different voltages between 0 and $2 \mathrm{~V}$ (see main panel in Figure 2). Interestingly, the slope of these curves grows (and hence the resistivity) as the voltage is increased. For example, the resistivity at $2 \mathrm{~V}, \rho_{\text {high }}$, is $320 \mathrm{k} \Omega / \mu \mathrm{m}$. If we repeat this analysis for the seven metallic nanotubes grown by CVD we find an average resistivity $\left\langle\rho_{\text {high }}\right\rangle=$
$330 \pm 110 \mathrm{~K} \Omega / \mathrm{nm}$ at $2 \mathrm{~V}$. Remarkably, this $\rho_{\text {high }}$ is quite similar for both CVD-grown and HipCo-type nanotubes. ${ }^{17}$ This result indicates that, for short nanotubes and high biases, inelastic scattering with optical phonons dominates over the elastic scattering with lattice defects.

The previous discussion shows that our experimental data for short nanotubes (shorter than $1 \mu \mathrm{m}$ ) closely reproduce the previously reported behavior for the electronic transport in SWNTs. ${ }^{9,10}$ However, when the distance between electrodes is longer than this length, $R(L)$ first exhibits a kind of saturation followed by a clear drop when the length of the nanotube is further increased (see main panel of Figure 2). We have obtained similar plots for the seven different metallic SWNTs considered in Figure 1, finding that the resistance saturation always takes place at SWNT lengths of about $1 \mu \mathrm{m}$. Therefore, we can safely conclude that this behavior is not a singularity of a given SWNT but the general trend appearing for very long nanotubes.

It has been suggested ${ }^{9,11}$ that the electron-phonon differential resistance of nonsuspended SWNTs can be described using a simple semiempirical approach whereby $R(L)$ $=\left(R_{0} / 2\right)\left(1+L / \lambda_{\text {eff }}\right), \lambda_{\text {eff }}$ being an effective mean free path (MFP) due to optical and acoustic phonons. $\lambda_{\text {eff }}$ is given by $1 / \lambda_{\text {eff }}=1 / \lambda_{\text {opt }}+1 / \lambda_{\text {ac }}$, where the MFPs of these phonons are introduced, and $\lambda_{\mathrm{opt}}=\lambda_{\mathrm{opt}}^{0}+\left(h v_{\mathrm{opt}} / \mathrm{eV}\right) \cdot L$, with $\lambda_{\mathrm{opt}}^{0}$ describing the optical MFP at zero $L, h v_{\text {opt }}$ being a mean optical phonon energy, and $V$ is the applied bias. The rationale behind this equation is that, at short SWNT lengths, the SWNT resistance is controlled by the number of optical phonons excited by one electron (that is assumed to be $e V /$ $h v_{\text {opt }}$ ), while at long lengths, electron scattering with acoustic phonons controls $R$. In panel c of Figure 2, we show the best fitting to our measured resistances using the above- 

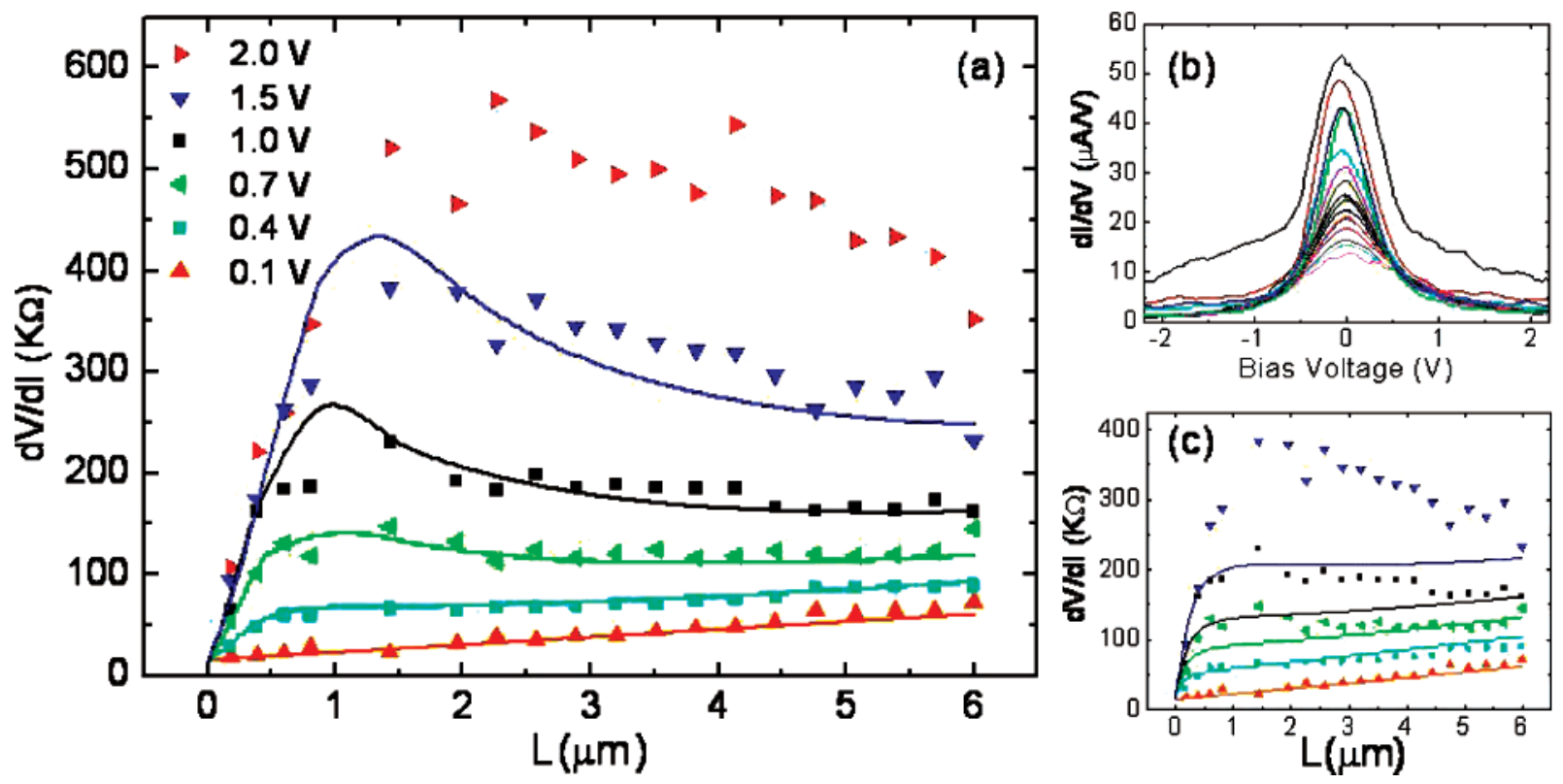

Figure 2. (a) Experimental dependence of the differential resistance, $\mathrm{d} V / \mathrm{d} I$, (in $\mathrm{K} \Omega$ ) vs length at different voltages for a single $6 \mu \mathrm{m}$ long CVD-SWNT (pink triangles in Figure 1). Full lines show the results of our theoretical modeling based on a discretised version of Boltzmann equations, using $\lambda_{\text {opt }}$ as a fitting parameter for each voltage, except for $V=0.1 \mathrm{~V}$ case (orange line) in which only scattering with acoustic phonons is taken into account. The set of data has been extracted from the family of differential conductances as a function of the voltage curves measured along the nanotube length panel (b). In panel (c), we render the results (full lines) of a fitting procedure based on a semiempirical theoretical approach.

mentioned formula, with $\lambda_{\mathrm{ac}}=650 \mathrm{~nm}$ and taking $\lambda_{\mathrm{opt}}^{0}$ as an adjustable parameter. Although the calculated resistances in this way show some of the trends found in our data, the fitting is rather poor, worsening at high voltages. Consequently, we have tried a more fundamental theoretical approach as explained in the following. This is a Monte Carlo solution of the one-dimensional Boltzmann's equation associated with the two conducting channels of the SWNT. In our semiclassical model, we consider that, for a given voltage (that must be smaller than $2 \mathrm{~V}$, in order to avoid higher electron bands contributing to transport), the energy window $E_{\mathrm{F} 1}-E_{\mathrm{F} 2}$ is discretized into $N$ levels, as shown in the panel b of Figure 3. Within our approach, electrons are injected into lead 1 and move along the tube at constant velocity toward lead 2. Electron-phonon processes scatter the electrons to lower energies as depicted schematically also in Figure 3b. Scattering with optical phonons is characterized by a mean free path, $\lambda_{\text {opt }}$, and a probability $\alpha$ of being scattered in the forward direction (the probability of backward scattering is then $(1-\alpha)$ ). In this way, forward and backward optical phonon scattering rates are given by $\alpha / \lambda_{\text {opt }}$ and $(1-\alpha) / \lambda_{\text {opt }}$, respectively. In our simulations, we use $\alpha$ $=0.25$, a value taken from independent local density approximation (LDA) calculations reported in ref 20 . We also incorporate into the theoretical modeling scattering of electrons with acoustic phonons. In this case, we only consider backward scattering, the main source of the SWNTresistance for quasi-elastic scattering, with a rate $1 / \lambda_{\mathrm{ac}}, \lambda_{\mathrm{ac}}$ being the electron mean free path due to scattering with acoustic phonons. We assume $\lambda_{\mathrm{ac}}=650 \mathrm{~nm}$, a value yielding the best fitting to the particular sample considered. The final probability rates per unit length $P_{i j}^{+,-}(x)$ for an electron at level $i$ moving in the forward direction to be scattered forward (+) or backward (-) to a final state $j$ need to be corrected by the occupancy numbers at the final level $j$, $n_{j}^{+,-}(x)$, leading to the following equation:

$$
\begin{aligned}
& \mathrm{d} P_{\mathrm{ij}}^{+}=\frac{\alpha}{\lambda_{\text {opt }}}\left[1-n_{j}^{+}(x)\right] \mathrm{d} x \\
& \mathrm{~d} P_{\mathrm{ij}}^{-}=\frac{1-\alpha}{\lambda_{\text {opt }}} \text { or } \frac{1}{\lambda_{\mathrm{ac}}}\left[1-n_{j}^{-}(x)\right] \mathrm{d} x
\end{aligned}
$$

with similar equations for electrons moving in the backward direction (-). The occupation numbers, $n_{j}^{+}(x)$ and $n_{j}^{-}(x)$, are calculated self-consistently by using a Monte Carlo approach whereby electrons are injected at each level and their trajectories are obtained introducing the different scattering processes defined by eqs $1-2$. In our Monte Carlo simulation, $m$ (of the order of thousands) electrons are injected at each level and contribute to the local densities, $n_{i}^{+}(x)$ and $n_{i}^{-}(x)$, with $1 / m$. An important point should be commented on: the problem presents electron-hole symmetry, with an electron at level $j$ of energy $E_{\mathrm{F} 1}-E$ behaving as a hole at level $k$ with energy $E_{\mathrm{F} 2}+E$. This symmetry leads to a neutrality condition in which $n_{j}^{+}(x)=1-n_{k}^{-}(L-$ $x)$ and $n_{j}^{-}(x)=1-n_{k}^{+}(L-x)$, showing that the total electron charge (sum of the forward and backward densities) of these two states ( $i$ and $j$ ) is 2 . Once the system is selfconsistently calculated and the local charge, $n(x)=\sum_{i}$ $\left[n_{i}^{+}(x)+n_{i}^{-}(x)\right]$, is obtained, we define the local chemical potential as:

$$
V(x)=\frac{E_{\mathrm{F} 1}+E_{\mathrm{F} 2}}{2}+\frac{E_{\mathrm{F} 1}-E_{\mathrm{F} 2}}{2}\left[\frac{n(x)}{N}-1\right]
$$



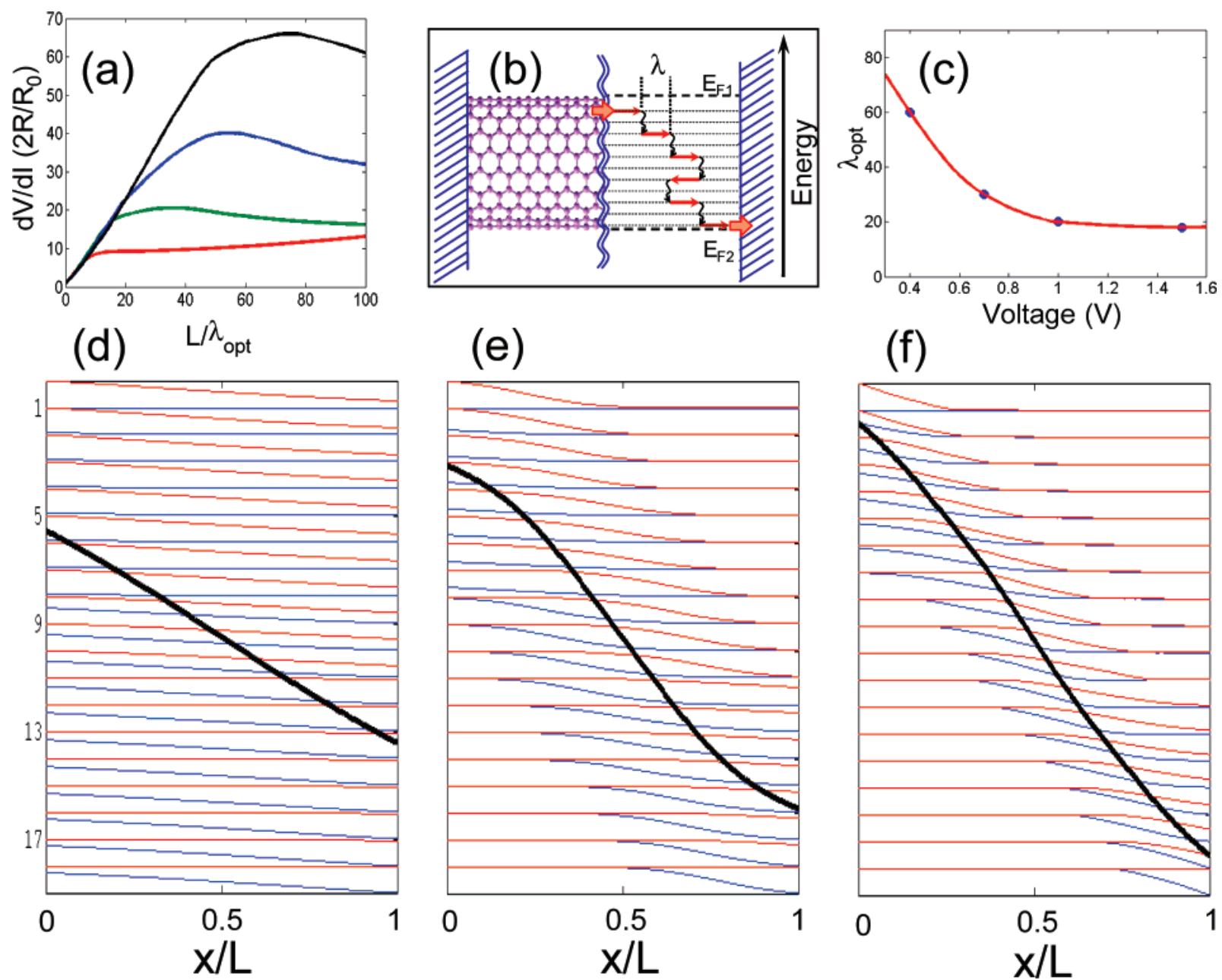

(e)

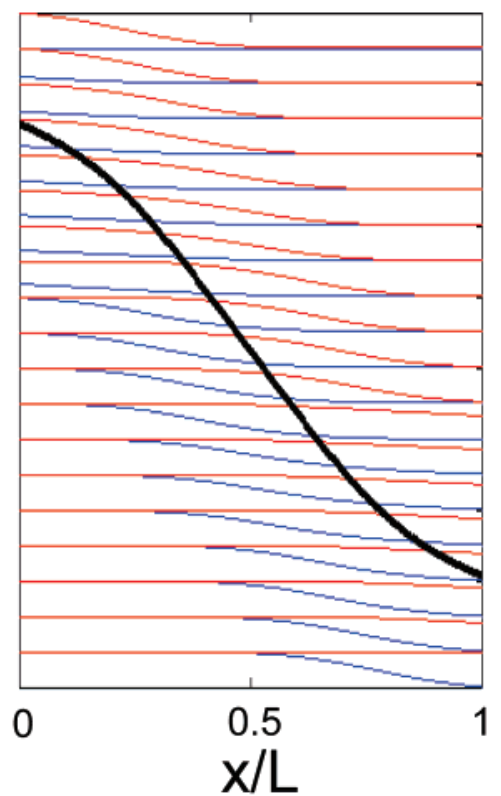

(f)

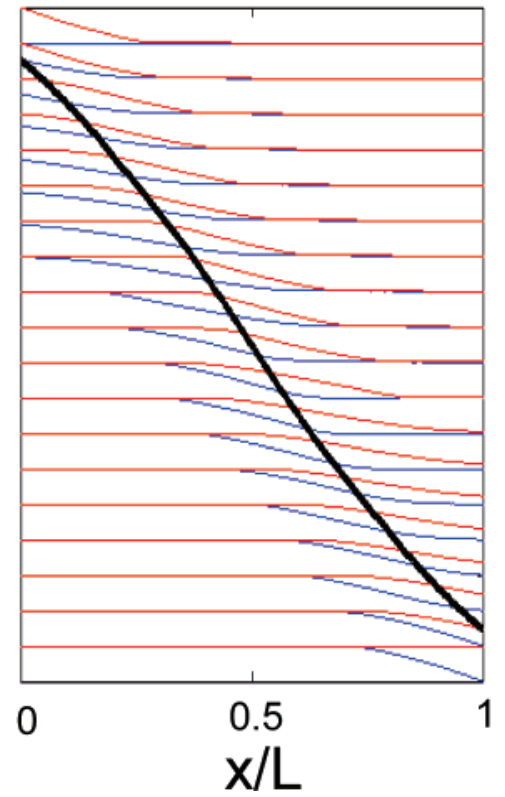

Figure 3. (a) Differential resistance $R$ (in units of the quantum of resistance) vs length of the nanotube $L$ (in units of $\lambda_{\text {opt }}$ ) obtained with our Monte Carlo theoretical approach for 4 different voltages: $0.4 \mathrm{~V}$ (red line), $0.7 \mathrm{~V}$ (green line), and $1.0 \mathrm{~V}$ (blue line), and $1.5 \mathrm{~V}$ (black line). (b) Schematic diagram to illustrate our theoretical modeling: the energy window $E_{\mathrm{F} 1}-E_{\mathrm{F} 2}$ is discretised into $N$-levels and electrons suffer scattering with acoustic and optical phonons characterized by $\lambda_{\mathrm{ac}}$ and $\lambda_{\text {opt }}$, respectively. (c) Dependence of $\lambda_{\text {opt }}$ vs the applied bias (in $\mathrm{V})$ as obtained from our calculations. Panels (d-f) render the dependence of $n_{i}^{+}(x)$ (red lines) and $n_{i}^{-}(x)$ (blue lines) vs $x$ (in units of the length of the nanotube) for $V=0.4 \mathrm{~V}$ ( $i$ ranging from 1 to 19). The curves corresponding to the $i-1$ level are shifted by -1 with respect to $i$-level curves for a better visualization. Three different lengths are analyzed: $L=92 \mathrm{~nm}$ (c), $L=480 \mathrm{~nm}(\mathrm{~d})$, and $L=6 \mu \mathrm{m}$ (e). Thick black lines render the local chemical potential $(V(x))$ for each of the three cases.

We assume that this chemical potential keeps the nanotube locally neutral. In our calculations, we first obtain the current for $N$ levels and, in order to calculate the differential conductance, $\sigma(R=1 / \sigma)$, we subtract the current for $(N-$ 1) levels and then divide by the energy difference between consecutive levels. We take this energy as $22.5 \mathrm{meV}$, close to $k_{\mathrm{B}} T$ for room temperature; in this way, we try to simulate the characteristic acoustic phonon energy involved in the electron-phonon scattering. Regarding optical phonons, we consider $h v_{\text {opt }}=180 \mathrm{meV},{ }^{21}$ eight times the acoustic phonon energy. In our numerical simulations, we analyze four different voltages, $0.4,0.7,1.0$, and $1.5 \mathrm{~V}$, and the corresponding $N$ for these cases is then $19,32,45$, and 64 , respectively.

In panel a of Figure 3 , we render $2 R / R_{0}\left(R_{0}\right.$ being the inverse of the quantum of conductance) as a function of $(L /$ $\lambda_{\text {opt }}$ ) for the four voltages studied. As clearly seen in this figure, in all these biases and small $L, 2 R / R_{0} \approx L / \lambda_{\text {opt }}$, showing that, in this limit, the optical phonons dominate the resistance behavior. As in the experimental data where the slope of $R / L$ depends on the applied bias, we conclude that $\lambda_{\text {opt }}$ is a function of voltage (as it is also the case in the semiempirical approach). The main panel of Figure 2 (full lines) shows our fitting to the experimental data by taking in our simulations: $\lambda_{\text {opt }}(0.4 \mathrm{~V})=55 \mathrm{~nm}, \lambda_{\text {opt }}(0.7 \mathrm{~V})=$ $30 \mathrm{~nm}, \lambda_{\text {opt }}(1.0 \mathrm{~V})=20 \mathrm{~nm}$, and $\lambda_{\text {opt }}(1.5 \mathrm{~V})=19 \mathrm{~nm}$. These values of $\lambda_{\text {opt }}$ can be understood in terms of the hot phonons created within the SWNT by the scattered electrons. Following the analysis described in ref 20, we can assume $\lambda_{\text {opt }}=\lambda_{\text {opt }}^{(0)} /\left(1+2 n_{\mathrm{B}}\right)$, where $\lambda_{\text {opt }}^{(0)}$ is the value without the effect of the optical phonon occupation number, $n_{\mathrm{B}}$. In this reference, a value of $88 \mathrm{~nm}$ for $\lambda_{\mathrm{opt}}^{(0)}$ has been calculated. In the limit of short SWNT lengths, $n_{\mathrm{B}}$ is expected to grow with the applied bias (because most of the excited phonons are optical). This suggests that $\lambda_{\text {opt }}$ should decrease with $V$, which is qualitatively what we obtain in our fitting (see Figure $3 \mathrm{c}$ ). For a more fundamental explanation of the 


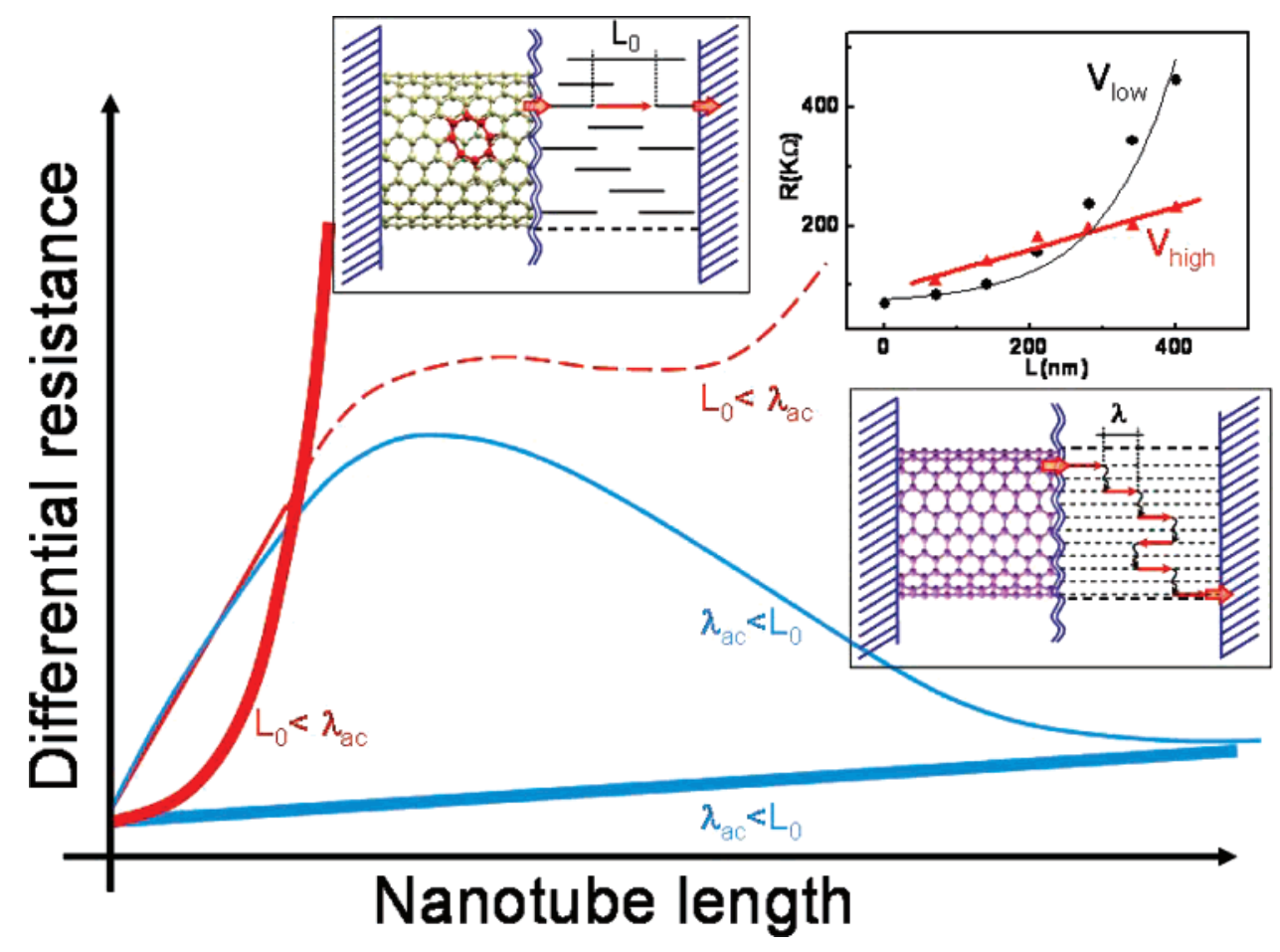

Figure 4. This figure summarizes the different electron transport regimes for SWNTs with and without defects as a function of the bias voltage and length. The thick lines apply to the low bias voltage. The thin lines apply for the high bias voltage. The blue curves are for defect-free SWNTs (lower inset) and the red ones for defected SWNTs (upper left inset). The upper right inset shows experimental $R(L)$ at low and high bias voltages for a defected SWNT whose localization length is $L_{0}=95 \mathrm{~nm}$.

dependence of $\lambda_{\text {opt }}$ with the applied bias, out-of-equilibrium optical phonons should be also included self-consistently within the transport equations by means of another semiclassical Boltzmann's equation for phonons ${ }^{20}$ (work along this line is currently in progress in our lab).

In Figure 3, panels $\mathrm{d}$, e, and $\mathrm{f}$ show the occupancy numbers, $n_{i}^{+}(x)$ (red lines) and $n_{i}^{-}(x)$ (blue lines), at each level and for $V=0.4 \mathrm{~V}$, evaluated at three different lengths, $L=92 \mathrm{~nm}, 460 \mathrm{~nm}$, and $6 \mu \mathrm{m}$, respectively. These values correspond to three different electronic transport regimes. For $L=92 \mathrm{~nm}$, the transport is still within the quasiballistic regime, with the chemical potential changing only slightly along the SWNT; notice that, in this case, $n_{i}^{-}(x)$ is different from zero only for levels $(i>9)$ where the optical phonons fill the backward direction states. For $L=460 \mathrm{~nm}, n^{-}(x)$ starts to be non-negligible even for $i<9$; this means that backward states are also filled in this case by acoustic phonon scattering processes. Now the chemical potential changes more quickly with a relatively small contact resistance. Finally, for $L=6 \mu \mathrm{m}$, the system has evolved in such a way that optical phonons play a minor role in the transport process: as shown in Figure 3f, most of the scattering events are controlled by the acoustic phonons, the reason being that, due to the small voltage gradient along the SWNT, the acoustic phonons have the opportunity to be more operative than the optical phonons.

This discussion shows that the resistance of the nanotube is controlled initially, for $0<L<1 \mu \mathrm{m}$, by the optical phonons; however, for $L>1 \mu \mathrm{m}$, the acoustic phonons start to play a more relevant role, being the predominant agent in a very long nanotube. In particular, for $V=0.4 \mathrm{~V}$, we find that around $L=6 \mu \mathrm{m}$, scattering of electrons with acoustic phonons mainly governs the resistance. This is confirmed by the experimental data, where $R$ is almost the same at $L$ $=6 \mu \mathrm{m}$ for low voltage and for $V=0.4 \mathrm{~V}$ (see Figure 2a). It is interesting to speculate about the expected behavior for higher biases. The previous discussion shows that the resistance, for high biases and $L>6 \mu \mathrm{m}$, should decrease slowly until reaching the "acoustic branch", rendered in Figure 2 by an orange line.

Finally, it is worth drawing a complete landscape of the electronic transport regimes in SWNTs by combining our findings for defect-free SWNTs with results for defected SWNTs. ${ }^{17}$ Figure 4 depicts a qualitative behavior of the SWNT-differential resistance as a function of its length for low (thick lines) and high (thin lines) voltages. As discussed above, for nondefected nanotubes (lower inset Figure 4 and blue curves), we can distinguish two regimes, depending on $e V$ being larger or smaller than $h v_{\text {opt }}$. For $e V<h v_{\text {opt }}$, the differential resistance shows a linear dependence (thick blue line) controlled by the scattering with acoustic phonons, $R=\left(R_{0} / 2\right)\left(1+L / \lambda_{\mathrm{ac}}\right)$. For $e V>h v_{\mathrm{opt}}$, optical phonons governs $R(L)$ for short nanotubes, $R=\left(R_{0} / 2\right)\left(1+L / \lambda_{\text {opt }}\right)$, while, at lengths about $1 \mu \mathrm{m}$, the differential resistance saturates and finally merges with the acoustic branch for very long nanotubes (thin blue line). Defected SWNTs (upper left inset and red curves) present an additional scattering mechanism characterized by the localization length, $L_{0}$, which is proportional to the average distance between defects. If $L_{0}$ $<L<L_{\varphi}$ ( $L_{\varphi}$ being the phase coherence length, determined by the electron-phonon mean free path, either $\lambda_{\mathrm{opt}}$ or $\lambda_{\mathrm{ac}}$ ), the SWNT-differential-resistance presents, at room temper- 
ature, ${ }^{18}$ an exponential dependence versus length, $R \propto e^{L / L_{0}}$, whatever the applied bias, typical of the strong Anderson localization regime. A more interesting case appears when $\lambda_{\text {opt }}<L_{0}<\lambda_{\mathrm{ac}}$; in this case, at low bias, electrons cannot excite optical phonons, $L_{\varphi} \approx \lambda_{\mathrm{ac}}$, then elastic scattering with defects prevails and the system becomes localized, the resistance exhibiting again the above commented exponential dependence with the nanotube length (thick red line), provided that the length of the nanotube is shorter than $\lambda_{\mathrm{ac}}$. At high biases, the electron energy is enough to scatter with optical phonons, $L_{\varphi} \approx \lambda_{\text {opt }}$, and because $L_{0}>\lambda_{\text {opt }}$, the system becomes diffusive, presenting a linear dependence for $R(L)$ for short nanotubes, controlled again by $\lambda_{\text {opt }}$ (thin red line). Experimental data of the transition between Anderson localization, at low bias, and diffusive transport, at high bias, on a short defected SWNT is depicted in the upper right inset of Figure 4. We do not have direct experimental evidence on the variation of the resistance for long lengths and high bias in defected nanotubes. However, based on our results, we expect that the elastic scattering of electrons with defects shall become the most effective scattering mechanism (notice that at those long lengths, the diffusive process is again dominated by acoustic phonons); then, as in the case $L_{0}<\lambda_{\mathrm{ac}}<L$, the nanotube conductance will be controlled by a thermally activated process ${ }^{22}$ due to acoustic phonons in the localized regime (thin red dashed line).

In conclusion, we have presented a detailed analysis on how the resistance of a SWNT evolves with its length. We have shown how the electrical transport regimes in SWNTs can be quasiballistic, localized, diffusive, and both diffusive and localized depending on the density of defects, length, and voltage. The phenomenology found in the electronic transport through SWNTs is more rich and complex than the one observed in classical conductors, and we have been able to capture it by means of a Monte Carlo approach to the one-dimensional Boltzmann's equation.

Acknowledgment. We acknowledge financial support from the Spanish CICYT under projects MAT2005-01298 and NAN-2004-09183-C10-07 and the Comunidad de Madrid/ Feder project under contract 07N/0050/2001.

\section{References}

(1) Iijima, S. Nature 1991, 354, 56-58.

(2) Tans, S. J.; Verschueren, A. R. M.; Dekker, C. Nature 1998, 393, 49-52.

(3) Ajayan, P. M.; Ebbesen, T. W. Rep. Prog. Phys. 1997, 60, 10251062.

(4) Baughman, R. H.; Zakhidov, A. A.; de Heer, W. A. Science 2002, 297, 787-792

(5) Bachtold, A.; Hadley, P.; Nakanishi, T.; Dekker, C. Science 2001, 294, 1317-1320.

(6) Javey, A.; Guo, J.; Wang, Q.; Lundstrom, M.; Dai, H. J. Nature 2003, 424, 654-657.

(7) Charlier, J.-C.; Blase, X.; Roche, S. Rev. Mod. Phys. 2007, 79, 677.

(8) Yao, Z.; Kane, C. L.; Dekker, C. Phys. Rev. Lett. 2000, 84, $2941-$ 2944.

(9) Park, J.-Y.; Rosenblatt, S.; Yaish, Y.; Sazonova, V.; Ustunel, H.; Braig, S.; Arias, T. A.; Brouwer, P. W.; McEuen, P. L. Nano Lett. 2004, 4, 517-520.

(10) Javey, A.; Guo, J.; Paulsson, M.; Wang, Q.; Mann, D.; Lundstrom, M.; Dai, H. Phys. Rev. Lett. 2004, 92, 106804.

(11) Pop, E.; Mann, D.; Cao, J.; Wang, Q.; Goodson, K.; Dai, H. J. Phys. Rev. Lett. 2005, 95, 155505.

(12) Horcas, I.; Fernández, R.; Gómez-Rodríguez, J. M.; Colchero, J.; Gómez-Herrero, J.; Baro, A. M. Rev. Sci. Instrum. 2007, 78, 013705/ $1-8$.

(13) Gomez-Navarro, C.; de Pablo, P. J.; Gomez-Herrero, J. Adv. Mater. 2004, 16, 549.

(14) de Pablo, P. J.; Gómez-Navarro, C.; Colchero, J.; Serena, P. A.; Gómez-Herrero, J.; Baró, A. M. Phys. Rev. Lett. 2002, 88, 036804

(15) Hafner, T. J. H.; Cheung, C. L.; Oosterkamp, T. H.; Lieber, C. M. High-yield fabrication of individual single-walled nanotube probe tips for atomic force microscopy. J. Phys. Chem. B 2001, 105, $743-$ 746.

(16) Pendry, J. B. Adv. Phys. 1994, 43, 461-542.

(17) Gómez-Navarro, C.; De Pablo, P. J.; Gómez-Herrero, J.; Biel, B.; Garcia-Vidal, F. J.; Rubio, A.; Flores, F. Nat. Mater. 2005, 4, 534539.

(18) Biel, B.; Garcia-Vidal, F. J.; Rubio, A.; Flores, F. Phys. Rev. Lett. 2005, 95, 266801.

(19) The mean free path due to acoustic phonons is probably underestimated due to the fact that it also includes, in an effective way, the quasi-elastic scattering of electrons with a very low density of defects present in our samples.

(20) Lazzeri, M.; Piscanec, S.; Mauri, F.; Ferrari, A. C.; Robertson, J. Phys. Rev. Lett. 2005, 95, 236802.

(21) This is an average of the energies of the optical phonon dispersion relation at $\Gamma$ and $\mathrm{K}$ points.

(22) Imry, Y. Introduction to Mesoscopic Physics; Oxford University Press: New York, 1998.

NL070746W 